\title{
Basis for paraxial surface-plasmon-polariton packets
}

\author{
Rosario Martinez-Herrero ${ }^{1}$ and Alejandro Manjavacas ${ }^{2, *}$ \\ ${ }^{1}$ Departamento de Óptica, Universidad Complutense de Madrid, 28040 Madrid, Spain \\ ${ }^{2}$ Department of Physics and Astronomy, University of New Mexico, Albuquerque, New Mexico 87131, USA
}

(Received 11 August 2016; published 15 December 2016)

\begin{abstract}
We present a theoretical framework for the study of surface-plasmon polariton (SPP) packets propagating along a lossy metal-dielectric interface within the paraxial approximation. Using a rigorous formulation based on the plane-wave spectrum formalism, we introduce a set of modes that constitute a complete basis set for the solutions of Maxwell's equations for a metal-dielectric interface in the paraxial approximation. The use of this set of modes allows us to fully analyze the evolution of the transversal structure of SPP packets beyond the single plane-wave approximation. As a paradigmatic example, we analyze the case of a Gaussian SPP mode, for which, exploiting the analogy with paraxial optical beams, we introduce a set of parameters that characterize its propagation.
\end{abstract}

DOI: 10.1103/PhysRevA.94.063829

\section{INTRODUCTION}

Surface-plasmon polaritons (SPPs) emerge from the mixing of electromagnetic fields and collective oscillations of conduction electrons near metal surfaces [1,2]. These excitations are capable of propagating along hundreds of microns at visible and near-infrared frequencies being, at the same time, confined in small transversal regions near the metallic surface [3-6]. Thanks to these properties, SPPs have become a versatile tool to guide and manipulate electromagnetic fields at length scales well below the diffraction limit of light [7-9], thus enabling applications as diverse as photonic interconnects [6,10-13] and ultrasensitive biosensors [14-16], to cite a few.

Although the fundamental properties of SPPs have been known for decades, there remain certain issues that can benefit from further analysis. Frequently, SPPs propagating at metal-dielectric interfaces are modeled using a single plane wave $[7,8,10]$. Even if this level of description may be sufficient in many situations, it ignores any possible transversal structure, thus missing, for instance, interesting cases such as nondiffractive solutions [17-20]. Therefore, a complete characterization of the evolution and the confinement properties of SPPs requires a description beyond the single plane-wave approximation [21-24] and a careful analysis of any simplification of the physical model since these can lead to incorrect outcomes [25-28].

The goal of this paper is to provide a theoretical framework for the study of SPP packets propagating at a lossy metaldielectric interface within the paraxial approximation. In order to do so, we employ a rigorous formulation based on the so-called plane-wave spectrum formalism [21,22,29,30], which takes into account explicitly the losses of the metal. By exploiting the analogy with the paraxial propagation of optical beams, we introduce a set of modes that constitutes a complete basis for the solutions of Maxwell's equations for a metal-dielectric interface in the paraxial approximation. Therefore, the propagation of an arbitrary SPP packet along the interface can be described using this set of modes, which allows us to fully analyze the corresponding evolution of its

*Corresponding author: manjavacas@unm.edu transversal structure beyond the single plane-wave description. Interestingly, the lowest-order mode of this set corresponds to a Gaussian SPP mode, which we analyze in detail as a paradigmatic example of a SPP packet. Furthermore, inspired by the analogy with the paraxial optical beams, we introduce the $q_{\mathrm{sp}}$ parameter, the radius of curvature, the intensity profile size, the Gouy phase, and the Rayleigh range, whose analysis serves to complete the characterization of the SPP packet evolution.

\section{GENERAL FORMULATION}

Our analysis starts by considering a flat interface perpendicular to the $z$ axis, which separates two homogeneous nonmagnetic media as shown in Fig. 1. The region below the interface (i.e., $z<0$ ) corresponds to a metallic medium characterized by a complex dielectric function $\varepsilon_{c}$ with $\operatorname{Re}\left\{\varepsilon_{c}\right\}<0$ and $\operatorname{Im}\left\{\varepsilon_{c}\right\}>0$. On the other hand, the region $z>0$ is filled with a dielectric medium whose dielectric function $\varepsilon_{d}$ is real and positive and satisfies $\varepsilon_{d}+\operatorname{Re}\left\{\varepsilon_{c}\right\}<0$. An electromagnetic wave of frequency $\omega$ propagates along the interface with an electric field in the dielectric medium given by

$$
\mathbf{E}_{d}(x, y, z)=\mathbf{E}_{0 d}(x, y) e^{i k_{d z} z} .
$$

Here, $k_{d z}^{2}=k_{\mathrm{sp}}^{2}\left(\varepsilon_{d} / \varepsilon_{c}\right)$, and $k_{\mathrm{sp}}^{2}=(\omega / c)^{2} \varepsilon_{c} \varepsilon_{d} /\left(\varepsilon_{c}+\varepsilon_{d}\right)$ is the SPP wave vector, which is a complex quantity for lossy metals. Interestingly, $k_{d z}$ is also complex even in the absence of losses, which ensures that the SPP is confined in the direction perpendicular to the interface. Since $\mathbf{E}_{d}$ satisfies Maxwell's equations and the corresponding boundary conditions, $\mathbf{E}_{0 d}$ must verify the two-dimensional Helmholtz equation,

$$
\frac{\partial^{2} \mathbf{E}_{0 d}}{\partial x^{2}}+\frac{\partial^{2} \mathbf{E}_{0 d}}{\partial y^{2}}+k_{\mathrm{sp}}^{2} \mathbf{E}_{0 d}=0,
$$

along with the transversality condition,

$$
\left(\frac{\partial}{\partial x}, \frac{\partial}{\partial y}, i k_{d z}\right) \cdot \mathbf{E}_{0 d}=0 .
$$

Using the angular plane-wave spectrum formalism of optical fields [29,30] as done in Ref. [23], it can be proven that, for $x \geqslant 0$, a solution of Eqs. (1) and (2) can be written as follows:

$$
\mathbf{E}_{0 d}(x, y)=\int_{-\infty}^{\infty} d u \tilde{\mathbf{E}}(u) e^{i x k_{x}(u)} e^{i y\left|k_{\mathrm{sp}}\right| u},
$$




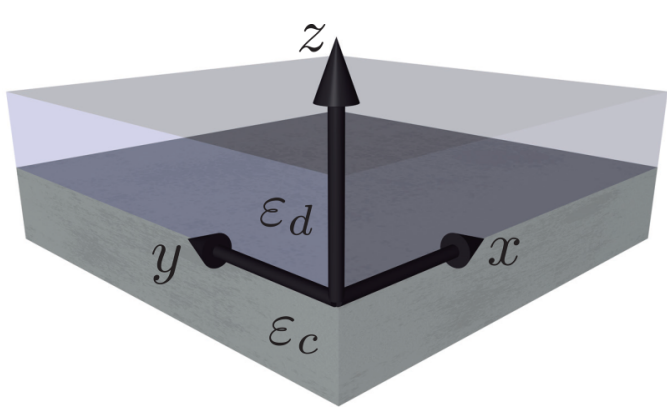

FIG. 1. Schematic of the system under study. We consider a flat interface perpendicular to the $z$ axis that separates a dielectric and a metallic medium with dielectric functions $\varepsilon_{d}$ and $\varepsilon_{c}$, respectively.

where

$$
\begin{aligned}
& \operatorname{Re}\left\{k_{x}(u)\right\}=\frac{1}{\sqrt{2}} \sqrt{\left|k_{x}^{2}(u)\right|+\operatorname{Re}\left\{k_{x}^{2}(u)\right\}}, \\
& \operatorname{Im}\left\{k_{x}(u)\right\}=\frac{1}{\sqrt{2}} \sqrt{\left|k_{x}^{2}(u)\right|-\operatorname{Re}\left\{k_{x}^{2}(u)\right\}},
\end{aligned}
$$

with $k_{x}^{2}(u)=k_{\mathrm{sp}}^{2}-\left|k_{\mathrm{sp}}^{2}\right| u^{2}$, and

$$
\tilde{\mathbf{E}}(u)=\left(k_{x}(u), u\left|k_{\mathrm{sp}}\right|,-k_{d z} \varepsilon_{c} / \varepsilon_{d}\right) F(u) .
$$

So that the components of the surface-plasmon field can be expressed as

$$
\mathbf{E}_{0 d}(x, y)=\left(-i \frac{\partial f}{\partial x},-i \frac{\partial f}{\partial y},-\frac{\varepsilon_{c} k_{d z}}{\varepsilon_{d}} f\right),
$$

where $f(x, y)$ is a scalar function defined as

$$
f(x, y)=\int_{-\infty}^{\infty} d u F(u) e^{i x k_{x}(u)} e^{i y\left|k_{\mathrm{sp}}\right| u} .
$$

Equation (3) describes a SPP packet, which physically can be understood as a superposition of inhomogeneous twodimensional fields with weights given by $F(u)$ that decay at different rates along the $x$ axis. Furthermore from Eq. (5) we see that the problem of SPP propagation at a metal-dielectric interface is polarization dependent and, therefore, strictly vectorial. However, we can always choose one of the components of the field and use it as a scalar potential from which all the other field components can be deduced. Therefore, without loss of generality, the problem can effectively be formulated in terms of the component of the electric field normal to the interface $f(x, y)$, which obeys the scalar Helmholtz equation,

$$
\frac{\partial^{2} f}{\partial x^{2}}+\frac{\partial^{2} f}{\partial y^{2}}+k_{\mathrm{sp}}^{2} f=0 .
$$

If we assume that the transverse profile of the SPP packet is slowly varying with respect to the propagation coordinate $x$, the function $F(u)$ has to be peaked around $u=0$, and therefore we can approximate $k_{x}(u)$ in Eq. (6) to obtain a simpler expression (see Appendix A),

$$
f(x, y)=e^{i x k_{\mathrm{sp}}} f_{0}(x, y),
$$

where

$$
f_{0}(x, y)=\int_{-\infty}^{\infty} d u F(u) \exp \left(-i x \frac{u^{2} k_{\mathrm{sp}}^{*}}{2}\right) e^{i y\left|k_{\mathrm{sp}}\right| u} .
$$

Here, the asterisk stands for complex conjugation. It is straightforward to show that $f_{0}$ satisfies the so-called paraxial equation,

$$
\frac{\partial^{2} f_{0}}{\partial y^{2}}+2 i k_{\mathrm{sp}} \frac{\partial f_{0}}{\partial x}=0 .
$$

It is important to notice that in this case $k_{\mathrm{sp}}$ is a complex quantity which leads, as will be seen below, to a number of important differences with respect to the well-known paraxial beam propagation in dielectric media.

\section{A BASIS FOR PARAXIAL SPP PACKETS}

Hermite-Gauss (HG) modes constitute a complete set of solutions of the paraxial wave equation for free space propagation [32]. The functions describing the transversal profile of HG modes are expressed, apart from normalization factors, as the product of a Hermite polynomial and a Gaussian function. Here, we exploit the analogy between the paraxial equation of optical beams and Eq. (8) to construct a basis set that is well suited to analyze the propagation for $x \geqslant 0$ of a SPP packet defined at $x=0$. Let us introduce the following set of functions:

$$
\begin{aligned}
u_{n}(x, y)= & A_{n} \sqrt{\frac{q_{0}}{q_{\mathrm{sp}}(x)}}\left(\frac{q_{0}-x}{q_{0}+x}\right)^{n / 2} H_{n}\left(\frac{y}{p(x)}\right) \\
& \times \exp \left(\frac{i y^{2} k_{\mathrm{sp}}}{2 q_{\mathrm{sp}}(x)}\right),
\end{aligned}
$$

where $A_{n}$ is a normalization constant, $q_{\mathrm{sp}}(x)=q_{0}+$ $x, p^{2}(x)=i\left(q_{0}^{2}-x^{2}\right) /\left(k_{\mathrm{sp}} q_{0}\right)$, and $q_{0}=-i k_{\mathrm{sp}} w_{0}^{2} / 2$. Here, $w_{0}^{2}$ is a real quantity that represents the Gaussian SPP width at $x=0$. When $k_{\mathrm{sp}}$ becomes real this set of functions reduces to the usual Hermite-Gauss modes. Furthermore, it can be proven that the functions $u_{n}(x, y)$ satisfy Eq. (8) and constitute, at $x=0$, a complete set of orthonormal functions characterized by a single complex parameter $q_{0}$. Therefore, they can be used as a basis set to expand any arbitrary $z$ component of the field of a SPP packet. In fact, given a certain $f(x, y)$ at $x=0$, we have $f(0, y)=f_{0}(0, y)$, and since $u_{n}(0, y)$ is a complete basis, we can write

$$
f_{0}(0, y)=\sum_{n=0}^{\infty} f_{0 n} u_{n}(0, y),
$$

where

$$
f_{0 n}=\int_{-\infty}^{\infty} d y f_{0}(0, y) u_{n}^{*}(0, y) .
$$

Then, using Eq. (7), we have that for any $x \geqslant 0$,

$$
f(x, y)=e^{i k_{\mathrm{sp}} x} \sum_{n=0}^{\infty} f_{0 n} u_{n}(x, y) .
$$

This allows us to write the complete expression for the propagation of the SPP packet as

$$
\begin{aligned}
& \mathbf{E}_{0 d}(x, y) \\
& \quad=e^{i k_{\mathrm{sp}} x} \sum_{n=0}^{\infty} f_{0 n}\left(-i \frac{\partial u_{n}}{\partial x}+k_{\mathrm{sp}} u_{n},-i \frac{\partial u_{n}}{\partial y},-\frac{\varepsilon_{c} k_{d z}}{\varepsilon_{d}} u_{n}\right) .
\end{aligned}
$$




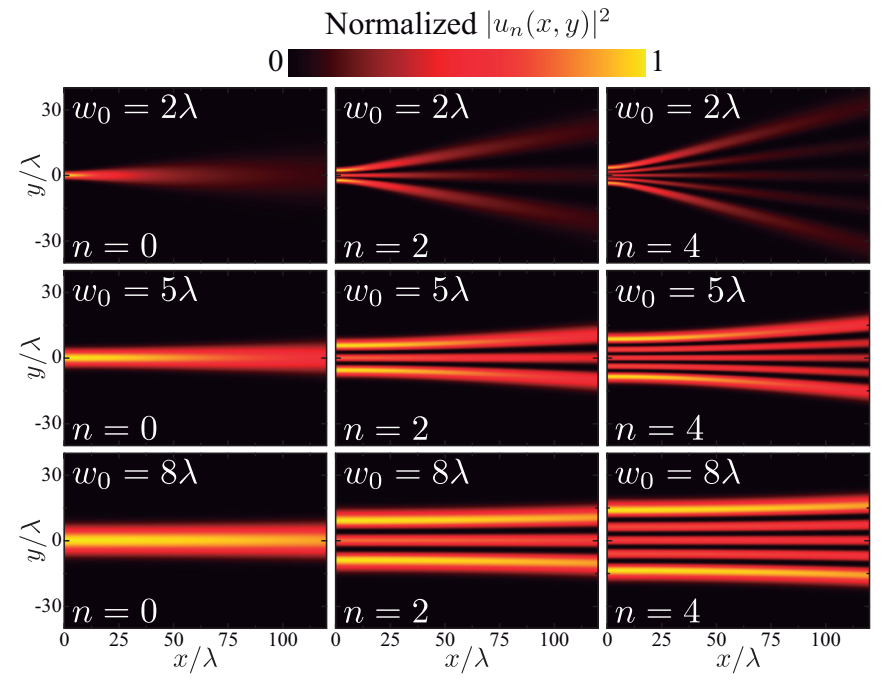

FIG. 2. Normalized square modulus of $u_{0}(x, y)$ (left column), $u_{2}(x, y)$ (center column), and $u_{4}(x, y)$ (right column) plotted for different values of $w_{0}: 2 \lambda$ (upper row), $5 \lambda$ (middle row), and $8 \lambda$ (bottom row). In all cases, we consider a silver-vacuum planar interface (i.e., $\varepsilon_{d}=1$ ) and choose the wavelength in vacuum to be $\lambda=633 \mathrm{~nm}$. The dielectric function of silver is taken from Ref. [31].

The coefficients $f_{0 n}$ depend on the arbitrary choice of $q_{0}$ at $x=0$. Therefore, in general, there is not a unique way of choosing the waist size for the basis set to expand a given SPP packet, similar to what happens in the case of free space propagating paraxial beams. We may attempt to choose this parameter in such a way that it results in the expansion that best fits the actual SPP field with the smaller number of terms. Figure 2 shows the square modulus of $u_{0}(x, y)$ (left column), $u_{2}(x, y)$ (center column), and $u_{4}(x, y)$ (right column) plotted for three different values of $w_{0}: 2 \lambda, 5 \lambda$, and $8 \lambda$, with $\lambda$ being the wavelength in vacuum, which we take equal to $633 \mathrm{~nm}$. We consider the case of a planar interface separating two half spaces made of silver and vacuum (i.e., $\varepsilon_{d}=1$ ). The dielectric function of the metal is obtained from the data tabulated in Ref. [31]. As expected, larger values of $n$ result in a more complex structure. Furthermore, the intensity profile spreads faster for smaller values of $w_{0}$, which is a consequence of the well-known trade-off between beam size and beam divergence for the paraxial propagation of beams.

As a direct consequence of the losses in the metal, $\operatorname{Im}\left\{k_{\mathrm{sp}}\right\} \neq 0$. This has two important implications: (i) It produces a global exponential decay for $\mathbf{E}_{0 d}(x, y)$ along the $x$ axis, characterized by a decay length $d_{x}=1 /\left(2 \operatorname{Im}\left\{k_{\mathrm{sp}}\right\}\right)$ [cf. Eqs. (5) and (7)], (ii) it makes the transversal intensity $I(x)$, defined as $I(x)=\int_{-\infty}^{\infty}\left|f_{0}(x, y)\right|^{2} d y$, dependent on $x$. Indeed, it can be proven that, within the paraxial approximation, the variation of $I(x)$ is given by (see Appendix B)

$$
\frac{d I(x)}{d x}=-\frac{\operatorname{Im}\left\{k_{\mathrm{sp}}\right\}}{\left|k_{\mathrm{sp}}\right|^{2}} \int_{-\infty}^{\infty}\left|\frac{\partial f_{0}(x, y)}{\partial y}\right|^{2} d y,
$$

and therefore, for finite $\operatorname{Im}\left\{k_{\mathrm{sp}}\right\}, I(x)$ decreases with $x$. This decay, which does not exist in the single plane-wave approximation, enhances the one caused by $e^{-x /\left(2 d_{x}\right)}$. Furthermore, as a consequence of the introduction of a complex argument in the Hermite polynomials, the propagation features of SPP packets differ substantially from those of the standard paraxial optical beams. In fact, the transversal intensity profile of the standard Hermite modes changes only by a scaling factor on propagation, whereas the transversal profile of modes given in Eq. (9) experience rather complicated changes.

\section{GAUSSIAN SPP MODE}

In order to get a deeper insight on the physical properties of the introduced basis we can consider the lowest-order mode from Eq. (9) (i.e., $n=0$ ), which corresponds to a Gaussian SPP mode,

$$
u_{0}(x, y)=A_{0} \sqrt{\frac{q_{0}}{q_{\mathrm{sp}}(x)}} \exp \left(\frac{i y^{2} k_{\mathrm{sp}}}{2 q_{\mathrm{sp}}(x)}\right) .
$$

By analogy with the well-known $q$ parameter of paraxial beams [30,32], we can introduce the real parameters,

$$
R_{\mathrm{sp}}(x)=\frac{\left|2 x-i k_{\mathrm{sp}} w_{0}^{2}\right|^{2}}{4 x},
$$

and

$$
w_{\mathrm{sp}}^{2}(x)=\frac{\left|2 x-i k_{\mathrm{sp}} w_{0}^{2}\right|^{2}}{2 x \operatorname{Im}\left\{k_{\mathrm{sp}}\right\}+\left|k_{\mathrm{sp}}\right|^{2} w_{0}^{2}} .
$$

According to these definitions the parameter $q_{\mathrm{sp}}(x)$ can be written as

$$
\frac{1}{q_{\mathrm{sp}}(x)}=\frac{\operatorname{Re}\left\{k_{\mathrm{sp}}\right\}}{k_{\mathrm{sp}} R_{\mathrm{sp}}(x)}+\frac{2 i}{k_{\mathrm{sp}} w_{\mathrm{sp}}^{2}(x)} .
$$

Notice that for a real $k_{\mathrm{sp}}$ this expression reduces to the usual $q$ parameter of paraxial beams. Taking into account the previous definitions, the lowest-order mode can be expressed as

$$
\begin{aligned}
u_{0}(x, y)= & A_{0}\left(\frac{\left|k_{\mathrm{sp}}\right| w_{0}^{2}}{\left|2 x-i k_{\mathrm{sp}} w_{0}^{2}\right|} \exp \left[-i \phi_{0}(x)\right]\right)^{1 / 2} \\
& \times \exp \left(\frac{i y^{2} \operatorname{Re}\left\{k_{\mathrm{sp}}\right\}}{2 R_{\mathrm{sp}}(x)}\right) \exp \left(-\frac{y^{2}}{w_{\mathrm{sp}}^{2}(x)}\right),
\end{aligned}
$$

where

$$
\tan \left[\phi_{0}(x)\right]=\frac{2 x \operatorname{Re}\left\{k_{\mathrm{sp}}\right\}}{2 x \operatorname{Im}\left\{k_{\mathrm{sp}}\right\}+\left|k_{\mathrm{sp}}\right|^{2} w_{0}^{2}} .
$$

Examining the expression for $u_{0}(x, y)$, we observe that it has the following desirable physical properties: (i) Its amplitude decays as the distance from the $x$ axis (i.e., $y$ ) increases, (ii) it carries a finite total power along the $y$ axis, and (iii) it keeps a Gaussian profile for $x \geqslant 0$ [see Fig. (2)]. The parameter $R_{\mathrm{sp}}(x)$ can be understood as the paraxial approach to the radius of curvature of a uniform spherical wave diverging from a point source located at the origin (i.e., $x=y=0$ ) of a medium with wave-number $\operatorname{Re}\left\{k_{\mathrm{sp}}\right\}$. Apart from this phase, the propagation with $x$ involves another phase given by $\phi_{0}(x)$. This axial phase is analogous to the well-known Gouy phase of paraxial beams [32]. On the other hand, $w_{\mathrm{sp}}^{2}(x)$ is the size of the intensity profile at $x$. As we can see from its definition, the evolution of this transversal size with $x$ is more involved than in the paraxial case, but, as expected, it 


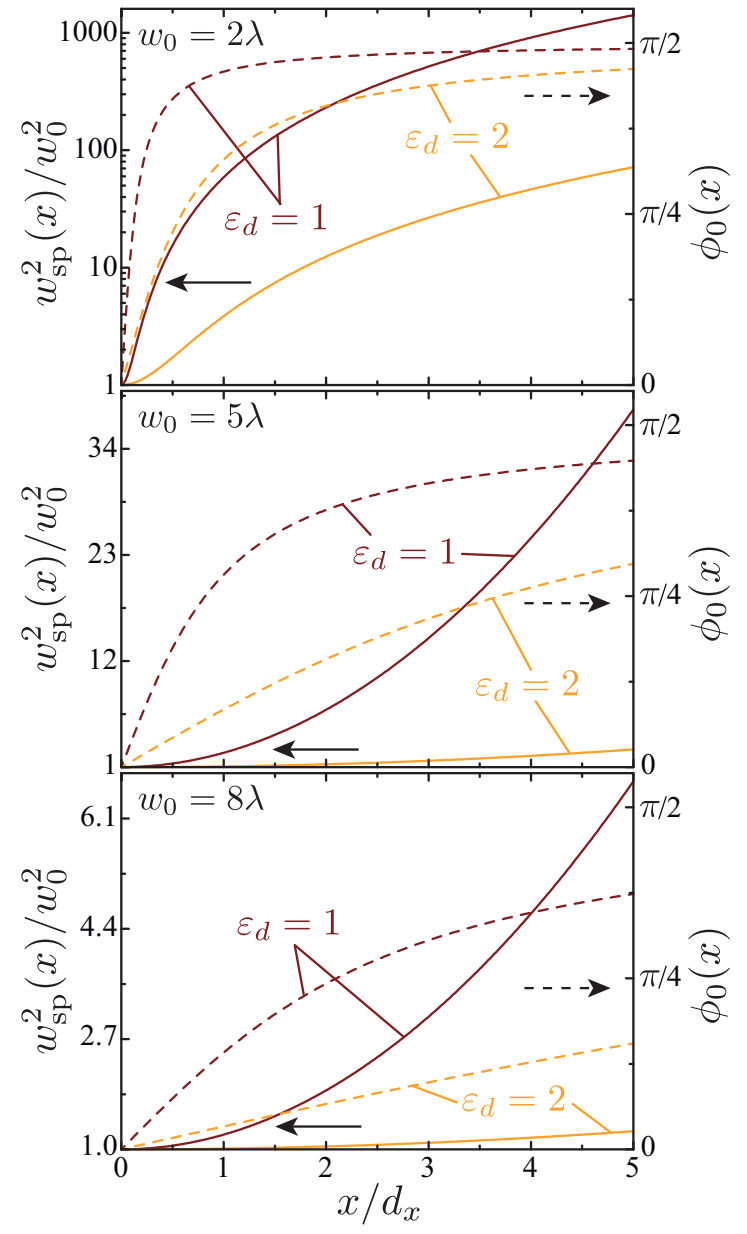

FIG. 3. Transversal size of the SPP packet $w_{\mathrm{sp}}^{2}(x)$ (solid lines, left scale) and Gouy phase $\phi_{0}(x)$ (dashed lines, right scale) plotted as a function of $x / d_{x}$ for different values of $w_{0}: 2 \lambda$ (upper panel), $5 \lambda$ (middle panel), and $8 \lambda$ (bottom panel). The wavelength in vacuum is $\lambda=633 \mathrm{~nm}$. In all cases, we consider a planar interface separating silver and a dielectric medium with $\varepsilon_{d}=1$ (dark red lines) and $\varepsilon_{d}=2$ (light yellow lines). The dielectric function of silver is taken from Ref. [31].

recovers the typical quadratic evolution when $k_{\mathrm{sp}}$ is a real quantity. Moreover, for a distance $x_{0}=\left|k_{\mathrm{sp}}\right| w_{0}^{2} / 2$, we have that $w_{\mathrm{sp}}^{2}\left(x_{0}\right)=2 w_{\mathrm{sp}}^{2}(0)=2 w_{0}^{2}$, which means that $x_{0}$ is the equivalent of the Rayleigh range [32]. At this point it is necessary to remember that the complete form of the mode is affected by an exponential decay along the $x$ axis [see Eq. (10)], which is determined by the dielectric constants of the involved media. Then, the ratio $x_{0} / d_{x}$ can be used as a measure of the broadening of a SPP packet propagating along a given metal-dielectric interface.

The behavior of $w_{\mathrm{sp}}^{2}(x)$ and $\phi_{0}(x)$ as functions of $x / d_{x}$ is illustrated in Fig. 3 for a planar interface separating silver and a dielectric medium with $\varepsilon_{d}=1$ (dark red lines) and $\varepsilon_{d}=2$ (light yellow lines). As in Fig. 2 we choose the wavelength in vacuum $\lambda$ to be $633 \mathrm{~nm}$. The solid lines and the left scale correspond to $w_{\mathrm{sp}}^{2}(x)$ normalized to $w_{0}^{2}$, whereas $\phi_{0}(x)$ is plotted using dashed lines and the right scale. These two quantities are calculated for three different values of $w_{0}: 2 \lambda$ (upper panel), $5 \lambda$ (middle panel), and $8 \lambda$ (bottom panel).
Examining the three panels of Fig. 3, we observe that the intensity profile size increases faster for smaller values of $w_{0}$, which is consistent with the discussion of Fig. 2. Similarly, larger values of $\varepsilon_{d}$ produce more confined SPP packets. On the other hand, the Gouy phase changes from 0 at $x=0$ to $\pi / 2$ as the SPP packet propagates. Interestingly, this process happens faster for smaller values of $w_{0}$ and $\varepsilon_{d}$. For higher-order modes the expression of $q_{\text {sp }}$ given in Eq. (12) remains useful, but for $n \geqslant 2$ there are significant differences with the Gaussian SPP mode. The complex argument in the Hermite polynomials gives an additional phase variation that modifies the spherical phase variation given by $R_{\mathrm{sp}}(x)$.

\section{CONCLUDING REMARKS}

To summarize, we have presented a theoretical framework to analyze the propagation of SPP packets along a lossy metal-dielectric interface within the paraxial approximation. Starting from the plane-wave spectrum, we have introduced a set of modes that is exceptionally well suited to describe the evolution of the transversal structure of SPP packets as they travel along the interface. Furthermore, by exploiting the analogy with the paraxial optical beams, we have adapted several parameters frequently used in that field to characterize SPP packets, namely, the $q_{\text {sp }}$ parameter, the radius of curvature $R_{\mathrm{sp}}$, the transversal size $w_{\mathrm{sp}}^{2}$, the Gouy phase $\phi_{0}$, and the Rayleigh range $x_{0}$. Incidentally, due to the complex character of $k_{\mathrm{sp}}$, the evolution of these parameters with the propagation differs strongly from the usual case of paraxial optical beams. The work presented here brings a different point of view to the characterization of the properties of SPP packets beyond the single plane-wave approximation and, therefore, is expected to trigger the development of new applications exploiting the exceptional properties of these excitations.

\section{ACKNOWLEDGMENTS}

This work has been sponsored by the Ministerio de Ciencia e Innovación (Spain) Projects No. FIS2013-46475 and No. FIS2016-75147 and the Department of Physics and Astronomy and the College of Arts and Sciences of the University of New Mexico. The authors also acknowledge the UNM Center for Advanced Research Computing for the computational resources used in this work.

\section{APPENDIX A: $k_{x}(u)$ IN THE PARAXIAL APPROXIMATION}

In the limit of $u \ll 1$, the real and imaginary parts of $k_{x}(u)$ given in Eq. (4) can be approximated by the following Taylor expansions:

$$
\begin{aligned}
& \operatorname{Re}\left\{k_{x}(u)\right\} \approx \operatorname{Re}\left\{k_{\mathrm{sp}}\right\}\left[1-\frac{u^{2}}{2}\right], \\
& \operatorname{Im}\left\{k_{x}(u)\right\} \approx \operatorname{Im}\left\{k_{\mathrm{sp}}\right\}\left[1+\frac{u^{2}}{2}\right],
\end{aligned}
$$

which combined produce $k_{x}(u) \approx k_{\mathrm{sp}}-k_{\mathrm{sp}}^{*} \frac{u^{2}}{2}$. 


\section{APPENDIX B: DERIVATION OF EQ. (11)}

Starting from Eq. (8) and multiplying by $f_{0}^{*} k_{\mathrm{sp}}^{*}$, we obtain

$$
f_{0}^{*} k_{\mathrm{sp}}^{*} \frac{\partial^{2} f_{0}}{\partial y^{2}}+2 i\left|k_{\mathrm{sp}}\right|^{2} f_{0}^{*} \frac{\partial f_{0}}{\partial x}=0
$$

whose complex conjugate is

$$
f_{0} k_{\mathrm{sp}} \frac{\partial^{2} f_{0}^{*}}{\partial y^{2}}-2 i\left|k_{\mathrm{sp}}\right|^{2} f_{0} \frac{\partial f_{0}^{*}}{\partial x}=0 .
$$

Now subtracting Eq. (B2) from Eq. (B1), we have

$$
2 i\left|k_{\mathrm{sp}}\right|^{2} \frac{\partial\left|f_{0}\right|^{2}}{\partial x}+f_{0}^{*} k_{\mathrm{sp}}^{*} \frac{\partial^{2} f_{0}}{\partial y^{2}}-f_{0} k_{\mathrm{sp}} \frac{\partial^{2} f_{0}^{*}}{\partial y^{2}}=0 .
$$

Finally, integrating over $y$ taking into account that $f_{0} \rightarrow 0$ for $|y| \rightarrow \infty$, we obtain

$$
2 i\left|k_{\mathrm{sp}}\right|^{2} \frac{d}{d x} \int\left|f_{0}\right|^{2} d y+\left(k_{\mathrm{sp}}-k_{\mathrm{sp}}^{*}\right) \int\left|\frac{\partial f_{0}}{\partial y}\right|^{2} d y=0 .
$$

[1] R. H. Ritchie, Phys. Rev. 106, 874 (1957).

[2] J. M. Pitarke, V. M. Silkin, E. V. Chulkov, and P. M. Echenique, Rep. Prog. Phys. 70, 1 (2007).

[3] W. L. Barnes, A. Dereux, and T. W. Ebbesen, Nature (London) 424, 824 (2003).

[4] E. Ozbay, Science 311, 189 (2006).

[5] R. Zia, J. A. Schuller, A. Chandran, and M. L. Brongersma, Mater. Today 9, 20 (2006).

[6] S. I. Bozhevolnyi, V. S. Volkov, E. Devaux, J. Y. Laluet, and T. W. Ebbesen, Nature (London) 440, 508 (2006).

[7] L. Novotny and B. Hecht, Principles of Nano-Optics (Cambridge University Press, New York, 2006).

[8] S. A. Maier, Plasmonics: Fundamentals and Applications (Springer, New York, 2007).

[9] I. V. Dzedolik and V. Pereskokov, J. Opt. Soc. Am. A 33, 1004 (2016).

[10] J. A. Dionne, L. A. Sweatlock, H. A. Atwater, and A. Polman, Phys. Rev. B 72, 075405 (2005).

[11] G. Veronis and S. Fan, Opt. Lett. 30, 3359 (2005).

[12] E. Moreno, S. G. Rodrigo, S. I. Bozhevolnyi, L. Martín-Moreno, and F. J. García-Vidal, Phys. Rev. Lett. 100, 023901 (2008).

[13] A. Manjavacas and F. J. García de Abajo, Opt. Express 17, 19401 (2009).

[14] C. E. H. Berger, T. A. M. Beumer, R. P. H. Kooyman, and J. Greve, Anal. Chem. 70, 703 (1998).

[15] J. Homola, S. Yee, and G. Gauglitz, Sens. Actuators, B 54, 3 (1999).

[16] M. A. Cooper, Nat. Rev. Drug. Discovery 1, 515 (2002).

[17] A. Salandrino and D. N. Christodoulides, Opt. Lett. 35, 2082 (2010).
[18] C. J. Zapata-Rodríguez, S. Vuković, M. R. Belić, D. Pastor, and J. J. Miret, Opt. Express 19, 19572 (2011).

[19] J. Lin, J. Dellinger, P. Genevet, B. Cluzel, F. de Fornel, and F. Capasso, Phys. Rev. Lett. 109, 093904 (2012).

[20] I. Epstein, R. Remez, Y. Tsur, and A. Arie, Optica 3, 15 (2016).

[21] A. Archambault, T. V. Teperik, F. Marquier, and J. J. Greffet, Phys. Rev. B 79, 195414 (2009).

[22] T. V. Teperik, A. Archambault, F. Marquier, and J. J. Greffet, Opt. Express 17, 17483 (2009).

[23] R. Martinez-Herrero, A. Garcia-Ruiz, and A. Manjavacas, Opt. Express 23, 28574 (2015).

[24] O. You, B. Bai, X. Wu, Z. Zhu, and Q. Wang, Opt. Lett. 40, 5486 (2015).

[25] A. R. Zakharian, J. V. Moloney, and M. Mansuripur, Opt. Express 15, 183 (2006).

[26] A. Norrman, T. Setälä, and A. T. Friberg, Opt. Lett. 38, 1119 (2013).

[27] A. Norrman, T. Setälä, and A. T. Friberg, Opt. Express 22, 4628 (2014).

[28] O. El Gawhary, A. J. L. Adam, and H. P. Urbach, Phys. Rev. A 89, 023834 (2014).

[29] R. Martínez-Herrero, P. M. Mejías, and A. Carnicer, Opt. Express 16, 2845 (2008).

[30] R. Martínez-Herrero, P. M. Mejías, and G. Piquero, Characterization of Partially Polarized Light Fields (Springer-Verlag, Berlin/Heidelberg, 2008).

[31] P. B. Johnson and R. W. Christy, Phys. Rev. B 6, 4370 (1972).

[32] A. Siegman, Lasers (University Science Books, South Orange, NJ, 1986). 